\title{
STELLAR KINEMATICS IN THE GALACTIC NORTH POLE REGION ON THE BASIS OF TAUTENBURG SCHMIDT TELESCOPE PLATES
}

\author{
N.V. KHARCHENKO ${ }^{1}$, E. SCHILBACH ${ }^{2}$ and R.-D. SCHOLZ ${ }^{2}$ \\ ${ }^{1}$ Main Astronomical Observatory of the Ukrainian Academy of Sciences, Kiev \\ ${ }^{2}$ WIP - Projekt Astrometrie bei der Universität Potsdam, Germany
}

\begin{abstract}
The spatial velocity components and their dispersions in the Galactocentric and rotation directions, eccentricities of Galactic orbits, parameters of spatial distribution and the change of all these characteristics with distance from the Galactic plane are determined. These data have been obtained on the basis of absolute proper motions and stellar $B, V$ magnitudes in two sky regions near the North Galactic Pole (NGP) by means of a plate set of the Tautenburg Schmidt telescope.
\end{abstract}

Investigations in the frame of the Galactic meridian programme (programme MEGA) have been carried out (Einasto et al. 1985) for the last ten years. MEGA includes 64 sky areas; of which 47 fields are the Kiev part and 17 fields are treated in Potsdam using plates obtained with the Schmidt telescope at Tautenburg. Kharchenko (1993) has already determined kinematical and spatial characteristics up to $2 \mathrm{kpc}$ on the basis of the Kiev fields. We have now begun the stellarstatistical study of the Galaxy up to $10 \mathrm{kpc}$ and more on the basis of Schmidt plates. We have selected two fields near the NGP; the 14th field of the Potsdam part of the MEGA programme (F14) and the field with the globular cluster M 3 (Table 1). In these fields we have determined the absolute proper motions of about 10,000 stars with respect to 3000 galaxies and their $B, V$ stellar magnitudes. The rms errors of these data are $\pm 3 \mathrm{mas} / \mathrm{yr}$ and $\pm 0.1 \mathrm{mag}$, respectively.

Table 1. Field characteristics

\begin{tabular}{lcc}
\hline & F 14 & M 3 \\
\hline \hline$\alpha_{1950.0}, \delta_{1950.0}$ & $12^{h} 48.8^{m},+30^{\circ} 28^{\prime}$ & $13^{h} 40.3^{m},+28^{\circ} 41^{\prime}$ \\
$l, b$ & $80^{\circ},+88^{\circ}$ & $42^{\circ},+79^{\circ}$ \\
area & 8.9 sq. degrees & 7.5 sq. degrees \\
$B_{l i m}$ & 19.0 & 20.5 \\
No. of plate pairs & 2 & 5 \\
measuring machine & MAMA , Paris & APM, Cambridge \\
\hline
\end{tabular}

The distances were determined by estimating absolute stellar magnitudes which were obtained on the basis of a joint analysis of the proper motions and reduced proper motions $H=V+5+5 \log \mu$, the colour-absolute magnitude diagrams of the Population I (Straizys 1977), 
the Population II (Sandage 1970) and compilated data of Chiu (1980). The value of the interstellar absorption near our fields was found to be $0.03-0.06$ mag from results of the spectral classification of Andruk et al. (1992). Figure 1 represents the reduced proper motion distribution in dependence on the $B-V$ colours for stars brighter than $B=18.3$ mag. According to Fig. 1 the majority of stars in the NGP have $B-V$ larger than 0.3 mag., i.e. practically all stars earlier than $F 0$ are evolved and leave the main sequence. There are only a few stars in the right upper corner, the region of red giants. Consequently, we observe stars either of the main sequence or subdwarfs or subgiants. There are a few white dwarfs in the left lower corner and a few stars of the blue part of the horizontal branch.

From the analysis of kinematical and spatial characteristics of stars in our fields, we have concluded that four subsystems can be distinguished in the NGP direction. These subsystems have spatial boundaries at $Z=0.3,1.0$ and $4.5 \mathrm{kpc}$ and age boundaries of $0.1,0.9$ and 0.95 of the Galaxy age. The age boundaries have been derived from the age calibration of the relationship between the rotation velocity around the Galactic $Z$-axis $v$ and velocity dispersion $\sigma$ (Einasto 1973). The semithicknesses are 0.15, 0.3, 0.65 and $1.5-2.5 \mathrm{kpc}$; the relations between $v$ and $\sigma[\mathrm{km} / \mathrm{s}]=2 v, 16.0-1.3 v, 53.2-0.4 v, 150$, respectively. The distributions of Galactic orbit eccentricities have been obtained for stars in different distance intervals. The eccentricities have been computed using the Galactic potential model of Saio \& Yoshii (1979) as well as velocity components. The estimation of velocity components in the $Z$ direction is based on the assumption that the stars move in flat elliptic inclinated orbits with the Galactic centre at their focus.

The data will be used for the development of Galactic models in a spatial volume comparable with the size of the Galaxy.

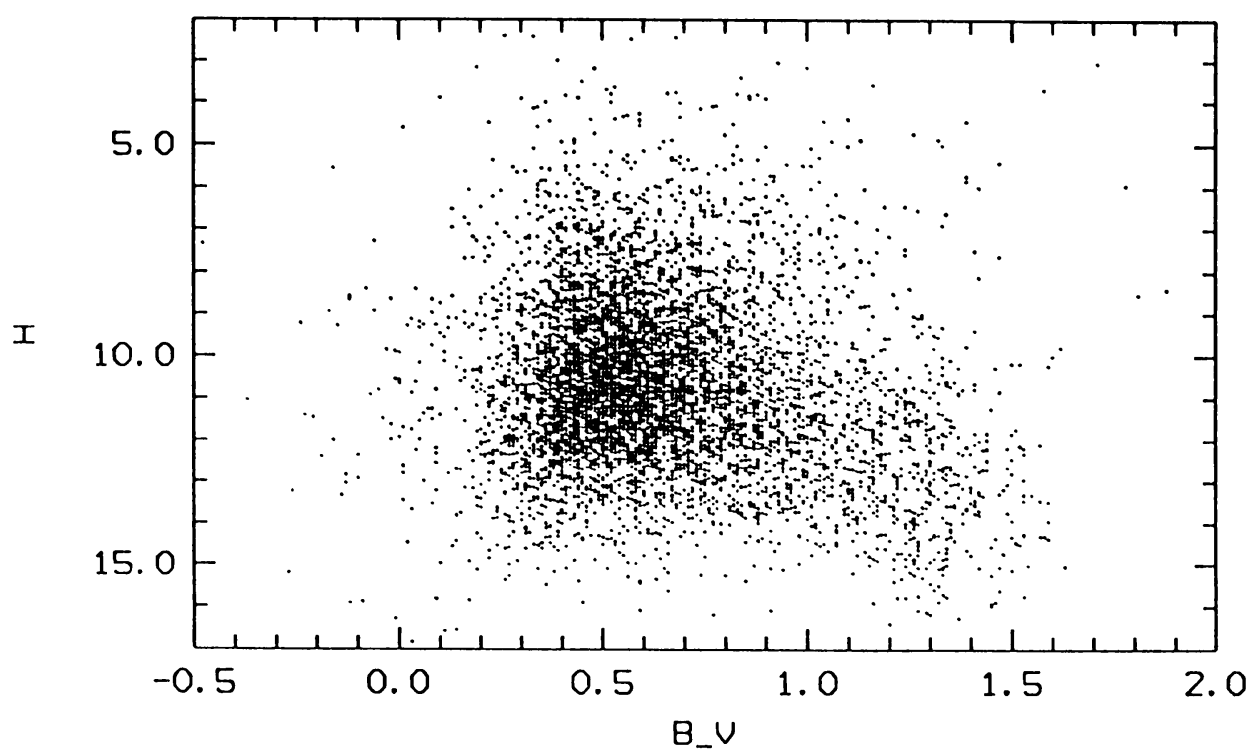

Figure 1. The reduced proper motion distribution as a function of $B-V$. 


\section{References}

Andruk, V.N., Bartashiute, S.A., Kharchenko, N.V., Malyuto, V.D. and Shvelidze, T.D., 1992. In 'The feedback of chemical evolution on the stellar content of galaxies', eds. D. Alloin and G. Stasinska, Observatoire de Paris, p. 189.

Chiu, L.-T.G., 1980. Astrophys. J., 44, 31.

Einasto, J., 1973. Astron. circular, 790, 3.

Einasto. J., Malyuto, V.D. and Kharchenko, N.V., 1985. Astron. circular, 1394, 1.

Kharchenko, N.V., 1993. In 'Developments in astrometry and their impact on astrophysics and geodynamics', eds. I.I. Mueller and B. Kolaczek, Kluwer Academic Publishers, Dordrecht, p. 231.

Saio, H. and Yoshii, Y., 1979. Publ. Astron. Soc. Pacif., 91, 553.

Sandage, A., 1970. Astrophys. J., 162, 841.

Straizys, V., 1977. 'Multicolour photometry of stars', Mokslas, Vilnius, p. 312. 